\title{
Methylation status of NEUROG2 and NID2 improves the diagnosis of stage I NSCLC
}

\author{
JUNFENG GENG $^{1 *}$, JINFENG SUN $^{2 *}$, QIANG LIN ${ }^{1}$, JUN GU$^{2}$, YANGXING ZHAO ${ }^{2}$, HONGYU ZHANG $^{2}$, \\ XU $\mathrm{FENG}^{3}$, YINGHUA HE${ }^{2}$, WEI WANG ${ }^{2}$, XIAOYU ZHOU ${ }^{4}$ and JIAN YU ${ }^{2}$ \\ ${ }^{1}$ Department of General Thoracic Surgery, Shanghai Chest Hospital, Shanghai Jiao Tong University, Shanghai 200030; \\ ${ }^{2}$ State Key Laboratory of Oncogenes and Related Genes, Shanghai Cancer Institute, Renji Hospital, \\ Shanghai Jiao Tong University School of Medicine, Shanghai 200032; ${ }^{3}$ Department of General Thoracic Surgery, \\ Guangxi Medical University, Tumor Hospital, Nanning 530022; ${ }^{4}$ Key Laboratory of Contraceptive Drugs and Devices, \\ National Population and Family Planning Commission, Shanghai Institute of Planned Parenthood Research, \\ Shanghai 200032, P.R. China
}

Received November 29, 2011; Accepted January 26, 2012

DOI: $10.3892 / \mathrm{ol} .2012 .587$

\begin{abstract}
In our previous study, we attempted to develop a tool for the early diagnosis of non-small cell lung cancer (NSCLC) using DNA methylation biomarkers. With the aim of improving the diagnostic potential by optimizing the composition of the target set, in this study, 13 candidate genes (ACTA1, AIDH1A2, CBX8, CDH8, EVX1, MGC16275, NEUROG1, NEUROG2, NID2, OTX2OS1, PGAM2, PHOX2B and TOX) were analyzed by methylation-specific PCR to determine the methylation status of each gene in 5 NSCLC cell lines and in lung tissue samples from 15 healthy volunteers, 103 stage I NSCLC patients and 26 non-cancerous control patients. Results showed that NEUROG2 and NID2 were hypermethylated in stage I NSCLC tissues (31.07 and 46.60\%, respectively) and unmethylated in normal lung tissues $(0 / 15)$ and non-cancerous tissues (0/26). Following recombination, an optimized 5-gene panel (NEUROG2, NID2, RASSF1A, APC and HOXC9) achieved a sensitivity of $91.26 \%$ with a specificity of $84.62 \%$ in the detection of stage I NSCLC. The optimized 5-gene panel greatly improved the diagnostic power for stage I NSCLC.
\end{abstract}

\section{Introduction}

Although the incidence and mortality rates for lung cancer have declined in the USA in the past decade, with the aging

Correspondence to: Dr Jian Yu, State Key Laboratory of Oncogenes and Related Genes, Shanghai Cancer Institute, Renji Hospital, Shanghai Jiao Tong University School of Medicine, LN 2200/25, Xietu Road, Shanghai 200032, P.R. China

E-mail: gyujian@163.com

*Contributed equally

Key words: NEUROG2, NID2, DNA methylation, non-small cell lung cancer, stage I of the population and rapid industrialization, the incidence of this malignancy has been on the increase in China where the mortality rate for lung cancer rose to the highest among all types of cancer (1). In addition to the toxic substances found in tobacco, a number of other chemical substances, including chromium, formaldehyde and nickel, can increase the risk of developing lung cancer (2). Non-small cell lung cancer (NSCLC), which accounts for $85 \%$ of all lung cancer cases, consists of three primary histological types: squamous cell carcinoma, adenocarcinoma and large cell carcinoma (3). The prognosis of NSCLC varies greatly depending on the clinical stage at diagnosis and the use of appropriate treatments. The overall 5-year survival rate is $73 \%$ for patients with pathological stage IA NSCLC, whereas this rate is only $25 \%$ for those patients with stage IIIA NSCLC $(4,5)$. Therefore, if biomarkers are established for the early detection and diagnosis of NSCLC, we may expect a marked decrease in mortality due to NSCLC.

Epigenetics is the study of mitotically and/or meiotically heritable changes in gene expression that are unrelated to changes in the DNA sequence (6). The dysregulation of epigenetic mechanisms is known to be involved in numerous diseases, including cancer (7). Epigenetic aberrations may occur at an earlier stage in tumor development than classical genetic transformation events, including mutations or deletions in tumor suppressor genes and proto-oncogenes. DNA methylation, a widely studied topic in epigenetics, was found to be abnormal in benign neoplasms and early stage tumors and both global DNA hypomethylation and promoter-specific hypermethylation have been observed. These attributes may qualify abnormal DNA methylation as a potential biomarker in the detection of cancer, especially at an early stage in carcinogenosis (8).

In our previous study, we identified a 5-gene panel (RASSF1A, APC, ESR1, HOXC9 and ABCB1) for the DNA methylation-based detection of stage I NSCLC with a sensitivity of $79.8 \%$ and a specificity of $73.1 \%$ for 124 cases of stage I NCSLC and 26 cases of non-cancerous respiratory disease (9). To further improve the diagnostic potential of this marker panel, in this study we screened more gene targets that 
Table I. Clinical profile of the stage I non-small cell cancer patients and controls.

\begin{tabular}{|c|c|c|c|}
\hline & $\begin{array}{l}\text { Non-small cell lung } \\
\text { cancer }(n=103)\end{array}$ & $\begin{array}{l}\text { Non-cancerous lung } \\
\text { lesions }(n=26)\end{array}$ & $\begin{array}{l}\text { Healthy volunteers } \\
\qquad(\mathrm{n}=15)\end{array}$ \\
\hline \multicolumn{4}{|l|}{ Gender } \\
\hline Female & 37 & 7 & 6 \\
\hline Male & 66 & 19 & 9 \\
\hline \multicolumn{4}{|l|}{ Age (years) } \\
\hline $31-40$ & 1 & 0 & 7 \\
\hline $41-50$ & 9 & 5 & 3 \\
\hline $51-60$ & 34 & 10 & 2 \\
\hline $61-70$ & 39 & 6 & 3 \\
\hline$\geq 71$ & 20 & 5 & \\
\hline Range & $32-79$ & $42-75$ & $23-66$ \\
\hline Median \pm SD & $61.13 \pm 9.12$ & $59.48 \pm 8.90$ & $42.47 \pm 16.78$ \\
\hline \multicolumn{4}{|l|}{ Stage/category } \\
\hline I & 103 & & \\
\hline Squamous & 25 & & \\
\hline Adenocarcinoma & 50 & & \\
\hline Adenosquamous & 13 & & \\
\hline Other & 15 & & \\
\hline \multicolumn{4}{|l|}{ Type of lung lesion } \\
\hline Pulmonary tuberculosis & & 6 & \\
\hline Bronchiectasis & & 4 & \\
\hline Pulmonary abscess & & 5 & \\
\hline Organizing pneumonia & & 2 & \\
\hline Pulmonary sclerosing hemangioma & & 3 & \\
\hline Pulmonary giant lymph node hyperplasia & & 1 & \\
\hline Pulmonary hamartoma & & 2 & \\
\hline Pulmonary sequestration & & 1 & \\
\hline Pulmonary inflammatory pseudotumor & & 2 & \\
\hline
\end{tabular}

are aberrantly methylated in NSCLC tissues and optimized the diagnostic panel. Since tumor-specific gene methylation may exist in different types of cancer $(10,11)$, we investigated 13 genes that were identified from data from a bladder methylation chip in our laboratory in NSCLC tissues. We found that the addition of 2 genes (NEUROG2 and NID2) to the diagnostic panel was of great benefit to the detection of stage I NSCLC.

\section{Materials and methods}

Cell lines and collection of the clinical tissue samples. The clinical tissue samples from 103 stage I NSCLC patients and 26 non-cancerous controls used in this study were the same as those used in our previous study and patient samples were obtained from the Shanghai Chest Hospital (Shanghai, China). Tumor-node-metastasis (TNM) staging/classification of the patient was performed according to the WHO classification (12). Normal lung tissue samples from 15 healthy organ donors were collected from the chest surgery department of the First Affiliated Hospital of Guangxi Medical University (Nanning, China) with the informed consent of the patients and the approval of the ethics committee. Table I shows the patient profiles, including the pathological types, ages and gender.

The lung cancer cell lines A549 [lung adenoma, ATCC (American Type Culture Collection, Manassas, VA, USA) no. CCL-185 ${ }^{\mathrm{TM}}$ ], NCI-H1299 (NSCLC, ATCC no. CRL-5803 ${ }^{\mathrm{TM}}$ ), LTEP-a-2 (lung adenoma, Cell Bank in Shanghai no. TCHu 33), SPC-A-1 (lung adenoma, Cell Bank in Shanghai no. TCHu 53) and NCI-H460 (large cell lung cancer, ATCC no. HTB-177 ${ }^{\mathrm{TM}}$ ) were cultured in L-DMEM medium containing $10 \% \mathrm{FBS}$ at $37^{\circ} \mathrm{C}$ in a $95 \%$ air, $5 \% \mathrm{CO}_{2}$ humidified incubator to the log phase of proliferation prior to the collection of the cells.

DNA isolation and methylation-specific PCR (MSP). Genomic DNA was isolated from the cell lines and the frozen clinical tissues, using conventional proteinase K/organic methods, as described in a previous study (13-15).

The primer pairs for MSP analysis used in this study were designed using software available online (http://www. urogene.org/methprimer/index1.html; Table II). The bisulfite conversion and PCR analyses were performed as previously 


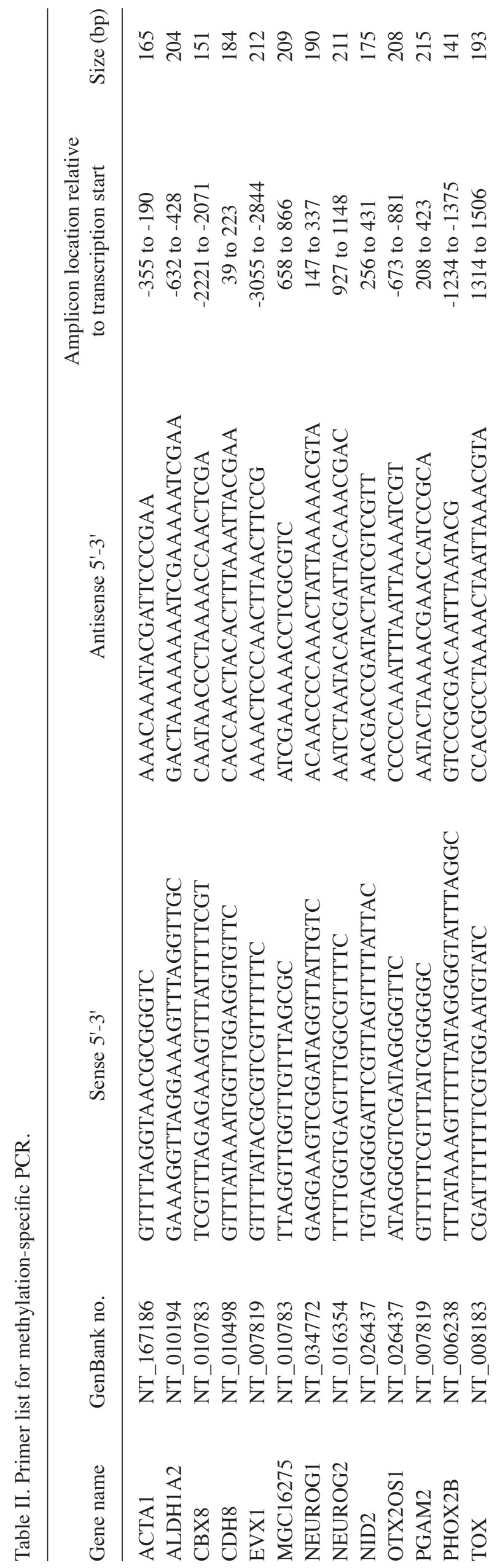

described $(13,15)$ and the representative PCR products were TA-cloned and then sequenced for validation.

Statistical analysis. Statistical calculations were performed with the use of SPSS 13.0 software. The incidence of hypermethylation in NSCLC tissues versus the non-cancerous tissues was calculated by a $2 \times 2$ Fisher's exact test. The associations among the pathological variables and the methylation status of the genes were assessed by means of univariate logistic-regression analysis. Multivariate regression models were then constructed to adjust for gender (female vs. male), smoking status (never smoked vs. smoker) and tumor histology.

The area under the receiver operating characteristics (ROC) curve (AUC) is a measure of the ability of a continuous marker to accurately classify tumor and non-tumor tissue. In this study, cut-off values were obtained to increase the sensitivity $[\%, \mathrm{TP} /(\mathrm{TP}+\mathrm{FN}),(\mathrm{TP}$, true positive; $\mathrm{FN}$, false negative $)]$ and the specificity $[\%, \mathrm{TN} /(\mathrm{TN}+\mathrm{FP}),(\mathrm{TN}$, true negative; FP, false positive)]. The sensitivity and the specificity of each gene set were calculated and plotted.

\section{Results}

MSP profiling of 13 genes in 5 NSCLC cell lines and 15 normal lung tissues. The 13 genes listed in Table II were analyzed by MSP to determine their methylation status in the $\mathrm{CpG}$ sites within their promoter or the first exon region. These genes were methylated in various NSCLC lines, ranging from 2 lines to all NSCLC cell lines (Table III).

In the 15 normal lung tissues, the methylation status of each gene ranged from 0 to $100 \%$. NEUROG2 and NID2 were unmethylated in all 15 normal lung tissues; by contrast, another 5 genes (CBX8, MGC16275, NEUROG1, PGAM2 and $\mathrm{PHOX} 2 \mathrm{~B}$ ) were methylated in all the normal lung tissues, while the remaining 6 genes [AIDH1A2 (20.00\%), ACTA1 $(33.33 \%)$, TOX $(40.00 \%)$, EVX1 (46.70\%), OTX2OS1 $(60.00 \%)$ and CDH8 (73.30\%)] were methylated in some of the normal lung tissues (Table III).

Consequently, we hypothesized that NEUROG2 and NID2 have the potential to discriminate lung cancer from healthy controls as these genes were methylated in several lung cancer cell lines but not in any of the normal lung tissues.

MSP profiling of NEUROG2 and NID2 in stage I NSCLC tissues and non-cancerous lesion controls. To further assess the potential of NEUROG2 and NID2 as biomarkers for the early detection of NSCLC, we MSP-profiled the two genes in the tissue samples from 103 stage I NSCLC patients and 26 patients suffering from non-cancerous lung disease; the cohorts were the same as those mentioned in our previous study (9). In this study, the methylation of NEUROG2 was detected in 32 of the $103(31.07 \%)$ cancer tissues but none $(0 \%)$ of the 26 non-cancerous control tissues, indicating a significant association between NEUROG 2 methylation and cancer. NID2 was methylated in 48 of the 103 (46.60\%) cancer tissues and unmethylated in all 26 non-cancerous control tissues; thus NID2 was also a cancer-specific gene (Table IV). Therefore, these two genes were demonstrated to be ideal biomarkers for the diagnosis of NSCLC. 
Table III. Methylation profile of lung cancer cell lines and healthy controls.

\begin{tabular}{lcccccc}
\hline Gene symbol & A549 & H460 & H1229 & LTEP-a-2 & SPC-A-1 & $\begin{array}{c}\text { Methylation frequency in } \\
\text { 15 healthy controls (\%) }\end{array}$ \\
\hline ACTA1 & 2 & 2 & 2 & 2 & 2 & 33.33 \\
AIDH1A2 & 1 & 2 & 2 & 2 & 2 & 20.00 \\
CBX8 & 2 & 2 & 2 & 2 & 2 & 100.00 \\
CDH8 & 2 & 2 & 2 & 2 & 2 & 73.30 \\
EVX1 & 2 & 2 & 2 & 2 & 2 & 46.70 \\
MGC16275 & 2 & 1 & 2 & 2 & 2 & 100.00 \\
NEUROG1 & 2 & 2 & 2 & 2 & 2 & 100.00 \\
NEUROG2 & 1 & 1 & 1 & 2 & 2 & 0.00 \\
NID2 & 1 & 1 & 2 & 2 & 2 & 0.00 \\
OTX2OS1 & 2 & 2 & 2 & 2 & 2 & 60.00 \\
PGAM2 & 2 & 2 & 2 & 2 & 2 & 100.00 \\
PHOX2B & 2 & 2 & 2 & 2 & 1 & 40.00 \\
TOX & 2 & 2 & 2 & 1 & &
\end{tabular}

1, unmethylated; 2 , methylated.

Table IV. The diagnostic performance of different panels of genes using patients with non-cancerous lung lesions as controls.

\begin{tabular}{lrrrrc}
\hline Gene symbol & Sensitivity, $\%$ & Specificity, $\%$ & PPV, $\%$ & NPV, $\%$ & P-value \\
\hline NEUROG2 & $31.07(32 / 103)$ & $100.00(0 / 26)$ & 100 & 26.8 & 0.0003 \\
NID2 & $46.60(48 / 103)$ & $100.00(1 / 26)$ & 100 & 32.1 & $<0.0001$ \\
NEUROG2, NID2, HOXC9 & $66.02(68 / 103)$ & $100.00(1 / 26)$ & 100 & 42.62 & $<0.0001$ \\
NEUROG2, NID2, RASSF1A, APC, ESR1, HOXC9, ABCB1 & $92.23(95 / 103)$ & $73.00(7 / 26)$ & 92.23 & 69.23 & $<0.0001$ \\
NEUROG2, NID2, RASSF1A, APC, HOXC9 & $91.26(94 / 103)$ & $84.62(5 / 26)$ & 95.92 & 70.97 & $<0.0001$ \\
RASSF1A, APC, ESR1, HOXC9, ABCB1 & $79.61(82 / 103)$ & $73.08(7 / 26)$ & 92.13 & 47.5 & $<0.0001$ \\
\hline
\end{tabular}

PPV, positive predictive value; NPV, negative predictive value.

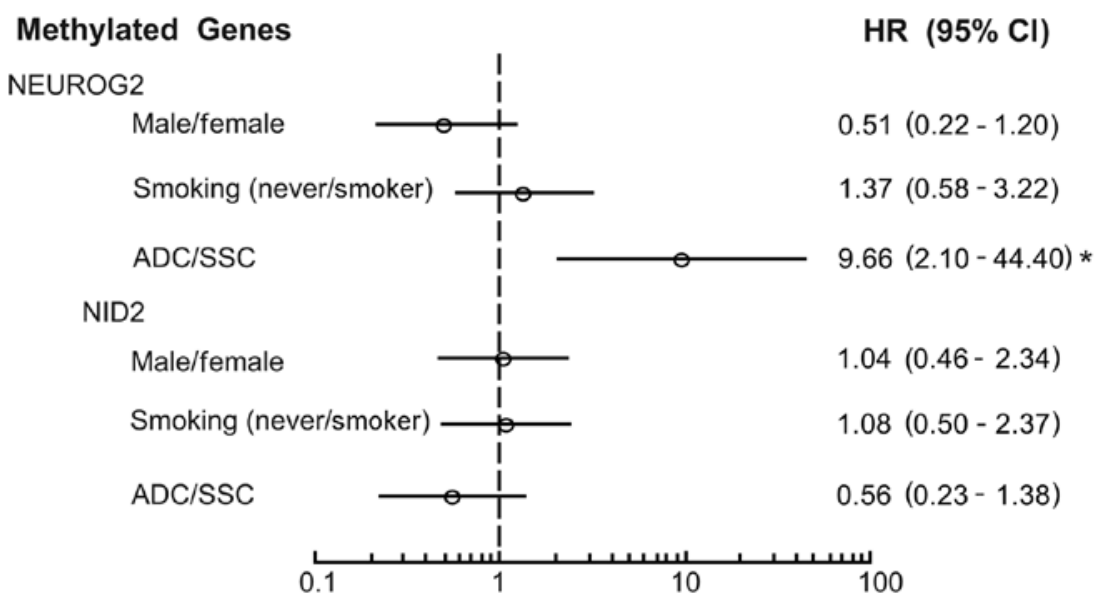

Figure 1. Associations of gene methylation (odds ratios) with pathological variables of NSCLC. Univariate logistic-regression analysis was used. HR, hazard ratio; CI, confidence interval; ADC, adenocarcinoma; SSC, squamous cell carcinoma; NSCLC, non-small cell lung cancer. *P<0.05.

Univariate analysis was carried out to assess the correlation between the methylation frequencies of NEUROG2 and NID2 with each of the clinical characteristics; the result was graphed as a forest plot. Notably, the methylation status of NEUROG2 differed greatly between ADC (adenocarcinoma) and SSC (squamous cell carcinoma) with an odds ratio of 9.66 (95\% CI 
Table V. Multivariate analysis of the tumor characteristics of NSCLC of different pathological types.

\begin{tabular}{llll}
\hline & HR & 95\% CI & P-value \\
\hline NEUROG2 (methylated vs. unmethylated) & 4.28 & $1.19-15.30$ & 0.0255 \\
Gender (male vs. female) & 0.03 & $0.00-0.25$ & 0.0013 \\
Smoking (never vs. smoker) & 1.28 & $0.42-3.90$ & 0.6623
\end{tabular}

HR, hazard ratio with 95\% CI, adjusted for multiple comparisons; CI, confidence interval; NSCLC, non-small cell lung cancer.

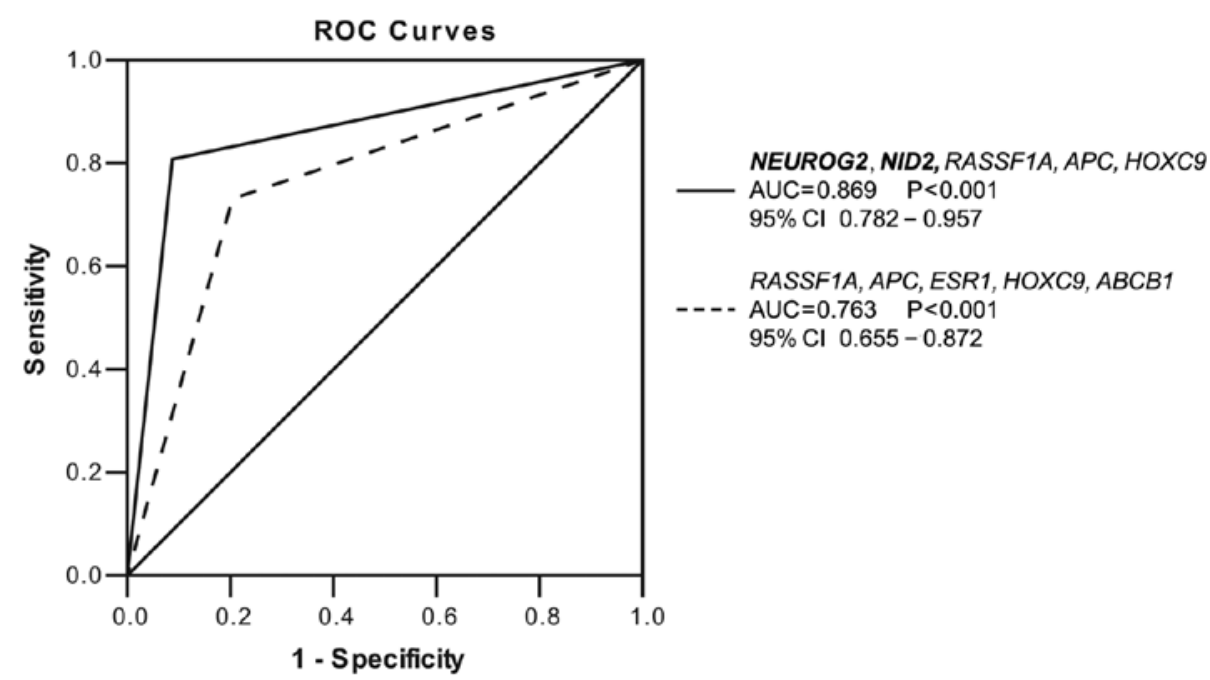

Figure 2. ROC curves for the two 5-gene panels. The curves show the capacity of the different panels of methylation markers to discriminate between NSCLC cases and controls. ROC, receiver operating characteristics; AUC, area under the ROC curve; NSCLC, non-small cell lung cancer.

2.10-44.40; P=0.0006; Fig. 1). Multivariate regression models were then established to consolidate the correlation between the methylation of NEUROG2 and ADC which was adjusted for other clinical characteristics, such as gender and smoking history. If NEUROG2 was methylated, the adjusted odds ratio for ADC was 4.28 (95\% CI 1.19-15.30; $\mathrm{P}=0.0255$; Table V).

No statistically significant correlation was observed between the methylation of either NEUROG2 or NID2 and either gender or smoking status.

By analyzing the ROCs, we found that the addition of NID2 to the previous panel of 5 genes (RASSF1A, APC, ESR1, HOXC9 and ABCB1) detected 11 additional stage I NSCLC patients, who were false negatives for the previous panel, thus improving the sensitivity from 79.61 to $90.29 \%$ with no decline in specificity. By adding another gene (NEUROG2), another 2 patients were diagnosed, raising the sensitivity to $92.23 \%$, and no additional false-positive cases were added. This combination of 7 genes (RASSF1A, APC, ESR1, HOXC9, ABCB1, NEUROG2 and NID2) provided $92.23 \%$ sensitivity and $73.08 \%$ specificity (Table IV).

However, to facilitate the screening for cancer in the general population, a panel of biomarkers should have a high specificity to minimize false positives; otherwise, numerous unnecessary follow-up procedures, which may be expensive and invasive, may be performed (16). A 3-gene panel (NEUROG2, NID2 and HOXC9) may provide 100\% specificity; however, the $66.02 \%$ sensitivity was barely satisfactory. To optimize the panel, we constructed another panel of 5 genes
(NEUROG2, NID2, RASSF1 A, APC and HOXC9), achieving a sensitivity of $91.26 \%$ and a specificity of $84.62 \%$ (Table IV).

ROC curves were constructed for each of the two panels of 5 DNA methylation biomarkers from our previous study (RASSF1A, APC, ESR1, HOXC9 and ABCB1) and the present study (NEUROG2, NID2, RASSF1A, APC and HOXC9). The ROC curve for the previous panel used to classify stage I NSCLC and non-cancerous lung disease yielded an AUC of 0.763 ( $\mathrm{P}<0.0001 ; 95 \%$ CI, 0.655-0.872); by contrast, the present panel yielded an AUC of $0.869(\mathrm{P}<0.0001 ; 95 \%$ CI, 0.782-0.957; Fig. 2).

The increase in the AUC demonstrates the superiority of the optimized panel of biomarkers.

\section{Discussion}

In our previous study, 5 genes (RASSF1A, APC, ESR1, HOXC9 and ABCB1) were combined as a panel for the diagnosis of stage I NCSLC by the method of MSP, which was able to achieve $79.8 \%$ sensitivity and $73.1 \%$ specificity, based on 124 cases of stage I NCSLC and 26 cases of non-cancerous respiratory disease (9).

Aiming to increase the sensitivity and specificity of DNA methylation as a biomarker for the diagnosis of NSCLC, we analyzed additional gene targets in this study in order to optimize the gene panel.

Recently, MBD-MethylCap/sequencing of bladder cancer cell lines and normal bladder mucosa was carried out in our 
laboratory, through which we identified numerous aberrantly methylated genes in bladder cancer cells (data unpublished). Aberrant methylation of $\mathrm{CpG}$ sites in the promoter region was found in more than one type of cancer and we selected 13 genes (ACTA1, AIDH1A2, CBX8, CDH8, EVX1, MGC16275, NEUROG1, NEUROG2, NID2, OTX2OS1, PGAM2, PHOX2B and TOX) from among the top 100 bladder cancer-specific genes to conduct further studies concerning the early detection and diagnosis of NSCLC. In this study, the tissue samples from the 103 stage I NSCLC patients and those from the 26 non-cancerous lung disease patients were from the same cohorts as used in our previous study, but the tissue DNA samples from some of the patients were no longer available.

The methylation of NEUROG2 and NID2 was associated with NSCLC. NEUROG2 and NID2 were found to be potential methylation biomarkers in stage I NSCLC diagnosis, being hypermethylated (NEUROG2, 31.07\%; NID2, 46.60\%) in cancer tissue samples but completely unmethylated in the non-cancerous controls.

Neurogenin-2, encoded by the NEUROG2 gene (4q25), is affiliated with the neurogenin subfamily of basic helix-loop-helix (bHLH) transcription factors, which are essential in neurogenesis from migratory neural crest cells (17). However, no evidence thus far has shown that NEUROG2 is correlated with the process of carcinogenesis.

The gene NID2 (1q43) encodes nidogen 1, which belongs to the nidogen family of basement membrane proteins and may play a role in maintaining the structure of the basement membrane (18). A number of studies have already reported that NID2 is correlated with cancer. The results of one study revealed that NID2 was methylated in $29 \%$ of colon cancer tissues and $95 \%$ of gastric cancer tissues, whereas it was completely unmethylated in the corresponding normal mucosa (19). Another study found that the concentration of nidogen-2 in the serum of patients suffering from ovarian cancer was elevated relative to that of normal controls and patients with benign conditions (20). At present, no report of an association between the methylation of NID2 and lung cancer is available.

This study was the first to demonstrate an association of these two genes with stage I NSCLC. Moreover, NEUROG2 showed an ADC-specific methylation.

An optimized 5-gene panel (NEUROG2, NID2, RASSF1A, APC and HOXC9) was developed for the early detection of NSCLC. Adding NEUROG2 and NID2 to the previous 5-gene panel markedly increased the sensitivity for stage I NSCLC diagnosis (from 79.61 to $92.23 \%$ ), but the $73.08 \%$ specificity of this 7-gene panel (RASSF1A, APC, ESR1, HOXC9, ABCB1, NID2 and NEUROG1) remained barely satisfactory. Removing two genes to yield another panel of 5 genes (NEUROG2, NID2, RASSF1A, APC and HOXC9) achieved a sensitivity of $91.26 \%$ with a specificity of $84.62 \%$. ROC analysis demonstrated that this optimized 5-gene panel was superior to the previous 5-gene panel and thus has greater potential for use in clinical diagnosis.

In conclusion, this study developed an optimized panel of methylation biomarkers for the diagnosis of stage I NSCLC. This panel needs further confirmation in a large patient cohort. Moreover, the gene NEUROG2 was reported to be associated with cancer for the first time and NID2 was studied in lung cancer for the first time. The involvement of these two genes in NSCLC should be explored further.

\section{Acknowledgements}

This study was supported in part by grants from the National Science Foundation (grant 30872963, J. Yu), the State Key Laboratory of Oncogenes and Related Genes (grant no. 91-11-01, J. Yu), the Shanghai Science Foundation (grant 09ZR1429900, Y. Zhao) and the Shanghai Cancer Institute Master \& Doctoral Foundation (SB09-07, Y. Zhao).

\section{References}

1. Chen W, Zhang S and Zou X: Estimation and projection of lung cancer incidence and mortality in China. Zhongguo Fei Ai Za Zhi 13: 488-493, 2010 (In Chinese).

2. Luo J, Hendryx M and Ducatman A: Association between six environmental chemicals and lung cancer incidence in the United States. J Environ Public Health: Jul 10, 2011 (Epub ahead of print).

3. Molina JR, Yang P, Cassivi SD, et al: Non-small cell lung cancer: epidemiology, risk factors, treatment, and survivorship. Mayo Clin Proc 83: 584-594, 2008.

4. Goldstraw P, Crowley J, Chansky K, et al: The IASLC Lung Cancer Staging Project: proposals for the revision of the TNM stage groupings in the forthcoming (seventh) edition of the TNM classification of malignant tumours. J Thorac Oncol 2: 706-714, 2007.

5. Besse B and Le Chevalier T: Adjuvant or induction cisplatin-based chemotherapy for operable lung cancer. Oncology (Williston Park) 23: 520-527, 2009.

6. Berger SL, Kouzarides T, Shiekhattar R and Shilatifard A: An operational definition of epigenetics. Genes Dev 23: 781-783, 2009.

7. Portela A and Esteller M: Epigenetic modifications and human disease. Nat Biotechnol 28: 1057-1068, 2010.

8. Feinberg AP: Cancer epigenetics is no Mickey Mouse. Cancer Cell 8: 267-268, 2005 .

9. Lin Q, Geng J, Ma K, et al: RASSF1A, APC, ESR1, ABCB1 and HOXC9, but not p16INK4A, DAPK1, PTEN and MT1G genes were frequently methylated in the stage I non-small cell lung cancer in China. J Cancer Res Clin Oncol 135: 1675-1684, 2009.

10. Shames DS, Girard L, Gao B, et al: A genome-wide screen for promoter methylation in lung cancer identifies novel methylation markers for multiple malignancies. PLoS Med 3: e486, 2006.

11. Chung W, Kwabi-Addo B, Ittmann M, et al: Identification of novel tumor markers in prostate, colon and breast cancer by unbiased methylation profiling. PLoS One 3: e2079, 2008.

12. Travis WD, Brambilla E, Müller-Hermelink HK and Harris CC (eds): World Health Organization Classification of Tumours. Pathology and Genetics of Tumours of the Lung, Pleura, Thymus and Heart. IARC Press, Lyon, 2004.

13. Yu J, Ni M, Xu J, et al: Methylation profiling of twenty promoter-CpG islands of genes which may contribute to hepatocellular carcinogenesis. BMC Cancer 2: 29, 2002.

14. Yu J, Zhang HY, Ma ZZ, et al: Methylation profiling of twenty four genes and the concordant methylation behaviours of nineteen genes that may contribute to hepatocellular carcinogenesis. Cell Res 13: 319-333, 2003.

15. Yu J, Zhu T, Wang Z, et al: A novel set of DNA methylation markers in urine sediments for sensitive/specific detection of bladder cancer. Clin Cancer Res 13: 7296-7304, 2007.

16. Ludwig JA and Weinstein JN: Biomarkers in cancer staging, prognosis and treatment selection. Nat Rev Cancer 5: 845-856, 2005.

17. Perrin FE, Boniface G, Serguera C, et al: Grafted human embryonic progenitors expressing neurogenin-2 stimulate axonal sprouting and improve motor recovery after severe spinal cord injury. PLoS One 5: e15914, 2010.

18. Kohfeldt E, Sasaki T, Göhring W and Timpl R: Nidogen-2: a new basement membrane protein with diverse binding properties. J Mol Biol 282: 99-109, 1998.

19. Ulazzi L, Sabbioni S, Miotto E, et al: Nidogen 1 and 2 gene promoters are aberrantly methylated in human gastrointestinal cancer. Mol Cancer 6: 17, 2007.

20. Kuk C, Gunawardana CG, Soosaipillai A, et al: Nidogen-2: a new serum biomarker for ovarian cancer. Clin Biochem 43: 355-361, 2010. 